gr-qc/0305072

VPI-IPPAP-03-07

CERN-TH/2003-109

\title{
Deconstructing the Cosmological Constant
}

\author{
Vishnu Jejjala, ${ }^{1 *}$ Robert G. Leigh, ${ }^{2,3 \dagger}$ and Djordje Minic ${ }^{1 \ddagger}$ \\ ${ }^{1}$ Institute for Particle Physics and Astrophysics \\ Physics Department, Virginia Tech \\ Blacksburg, VA 24061, U.S.A. \\ ${ }^{2}$ CERN-Theory Division \\ CH-1211, Geneva 23, Switzerland \\ ${ }^{3}$ Department of Physics \\ University of Illinois at Urbana-Champaign \\ 1110 W. Green Street, Urbana, IL 61801, U.S.A.
}

\begin{abstract}
Deconstruction provides a novel way of dealing with the notoriously difficult ultraviolet problems of four-dimensional gravity. This approach also naturally leads to a new perspective on the holographic principle, tying it to the fundamental requirements of unitarity and diffeomorphism invariance, as well as to a new viewpoint on the cosmological constant problem. The numerical smallness of the cosmological constant is implied by a unique combination of holography and supersymmetry, opening a new window into the fundamental physics of the vacuum.
\end{abstract}

*vishnu@vt.edu

${ }^{\dagger}$ rgleigh@uiuc.edu

${ }^{\ddagger}$ dminic@vt.edu 
The validity of general relativity as a classical theory, at least at reasonable length scales is by now beyond any doubt, yet a completely satisfying quantum theory of gravitation remains elusive. The difficulty may be understood on many levels. The most straightforward approach, that of treating general relativity as a local four-dimensional field theory and quantizing it as such, fails unequivocally. The gravitational coupling, $G_{N}$, is a dimensionful quantity that renders the short-distance structure of the theory meaningless. Thus, at best, general relativity should be regarded as a four-dimensional effective field theory that is replaced by something else at short distances, for example, a well-defined perturbative quantum theory of gravity, such as string theory.

Yet, all is not well, even apart from the basic open question of how to formulate a background independent non-perturbative version of quantum gravity. The low-energy effective field theory makes predictions wildly inconsistent with observation. Most notably, when coupled to matter degrees of freedom, the energy density of the vacuum is extremely large, scaling with the largest available energy in the theory. This is the essence of the cosmological constant problem. The insidiousness of the renormalization of the cosmological constant means that it is not even sufficient to find a principle that would set the vacuum energy to some small value at a given ultraviolet (UV) scale; rather it must be canceled all the way into the infrared (IR).

It has recently become clear that quantum gravitational systems display features that cannot be accommodated by local four-dimensional field theories. In particular, the holographic principle [1] asserts that the degrees of freedom of such four-dimensional gravitational systems are better accounted for by three-dimensional data. This principle stems from the well-known non-extensive properties of the Bekenstein-Hawking entropy [2]

$$
\mathcal{S}=\frac{A}{4 G_{N}}
$$

which scales as the area, not the volume, of a given region of space. Just how holography might be implemented is a matter of some debate, but simple examples, possessing a high degree of symmetry, have been well explored; this is what underlies the duality between gravitating systems on anti-de Sitter (AdS) background geometries and conformal field theories (CFT) in one fewer dimension 3 .

If holography is to be taken seriously, we should look to three-dimensional theories for guidance. Recent astrophysical observations of the cosmic microwave background radiation [4] and distant supernovae [5] together suggest that the expansion of the universe is accelerating and that this acceleration is being driven by a "dark energy," which comprises three quarters of the total energy density of the universe. The leading candidate for dark energy is the energy in the vacuum itself, and the observed value points to a positive small cosmological constant. An extension of the ideas underlying the dualities mentioned above would then seem to suggest looking for a de Sitter/CFT correspondence [6]. It is not clear however, what three-dimensional CFT would be capable of fully describing the present state of our Universe.

However, there is another possibility based on the idea of deconstruction [7. In this framework, one imagines that the short distance regime of a four-dimensional field theory is 
described by a three-dimensional theory. The most amazing possibility is that by introducing supersymmetry into the three-dimensional theory, it is possible that the four-dimensional theory has a small cosmological constant! This statement relies on specific properties of three-dimensional supersymmetry, first noticed by Witten [8]. Furthermore, there are signals that holography may be operating in this scenario, although in a much different guise than in $\mathrm{AdS} / \mathrm{CFT}$.

In deconstruction, an infrared theory is placed on a one-dimensional lattice. The link fields that connect adjacent lattice sites provide a Goldstone realization of an ultraviolet theory in one lower dimension. The continuum limit of the lattice theory dynamically generates an additional spatial direction in the infrared. Gravity can be studied within this formalism 19, 10, 11. Remarkably, it can be argued that a four-dimensional quantum theory of gravitation emerges as the infrared limit of coupled $(2+1)$-dimensional theories of gravity on a lattice [10, 11]. The Bekenstein-Hawking entropy formula (up to the purely numerical factor) is a universal statement about the mixing of the UV and IR physics, which violates the basic principles of a local effective field theory [9, 11].

More explicitly, assuming a local spatial foliation of spacetime, the Einstein-Hilbert action

$$
S=\frac{1}{G_{N}} \int \epsilon_{a b c d} e^{a} \wedge e^{b} \wedge R^{c d}
$$

expressed in terms of the vierbein and curvature, is classically the deconstructed version of $N$ copies of three-dimensional general relativity (a Chern-Simons theory) coupled to a set of three-dimensional currents [10. The parameters of the three-dimensional theory are regarded as fundamental. The four-dimensional Newton constant is a derived quantity that is determined by the three-dimensional Newton constant and the lattice spacing $a$ : $G_{N}=$ $1 / M_{P l}^{2}=G_{3} a$. Four-dimensional matter fields may also be defined in terms of a deconstructed three-dimensional theory [7].

In a perturbative quantum theory of gravity, the exchange of gravitons - local, propagating degrees of freedom - mediates the dynamical response of spacetime to the presence of energy and, conversely, the dynamical response of matter to the geometry of spacetime. However, in $2+1$ dimensions, gravity is purely topological [12]. There are no local degrees of freedom at all. To recover the local character of gravitational dynamics in $3+1$ dimensions, one needs the non-gravitational part of the ultraviolet completion. Indeed, "most" of fourdimensional gravity is reconstructed from the matter sector (the link fields) of the lattice realization. These are precisely the three-dimensional currents in our construction [10]. The infrared theory organizes this co-dimension one skeleton into the architecture of spacetime making four-dimensional Lorentz invariance an emergent property of the continuum limit.

One of the outstanding features of this construction is that it offers a new viewpoint on the cosmological constant problem [10, 11]. In the deep ultraviolet, there are $N$ essentially independent copies of three-dimensional gravity coupled to three-dimensional sources. These sources induce a conical geometry whose deficit angle prohibits spinor fields with covariantly constant asymptotics [13]. This means that unbroken global supercharges do not exist. We can have a supersymmetric vacuum without mass degenerate Bose/Fermi excitations [8]. Three-dimensional supersymmetry therefore implies that the vacuum energy exactly 
vanishes at each lattice site. In the range of intermediate scales, there are $N$ linked copies of three-dimensional gravity, now coupled to three-dimensional currents. The geometry is again conical, and the vacuum energy still vanishes. In the infrared, we recover fourdimensional general relativity with non-zero cosmological constant. This is the consequence of a gravitational see-saw, which balances the Planck mass against the infrared scale $\Delta m$ determined by the Bose/Fermi mass splitting [11].

The crucial observation here is that the infrared dynamics ties together intimately with the physics in the ultraviolet regime. A tree-level computation indicates that amplitudes involving the longitudinal components of gravitons de-unitarize at a scale [9, 11]

$$
\mu \sim\left(\frac{M_{P l}^{2}}{L^{5} a^{2}}\right)^{1 / 9}
$$

where $L=N a$ is the lattice size. By demanding that the theory is truncated above the most massive Kaluza-Klein states but below the unitarity threshold, we find that the maximum possible cutoff is of order

$$
\mu_{\text {max }} \sim\left(\frac{M_{P l}^{2}}{L}\right)^{1 / 3} .
$$

This exemplifies the phenomenon of UV/IR mixing: the ultraviolet cutoff is defined in terms of purely infrared quantities, namely the size of the extra spatial dimension that arises from deconstruction and the four-dimensional Planck mass. ${ }^{1}$

The scale $\mu_{\max }$ has an important holographic interpretation. We can compute the entropy using the thermodynamic relation $\mathcal{S} \sim V T^{3}$, where the volume $V \sim A L$ and the temperature $T \sim \Lambda_{\max }$, the ultraviolet cutoff. Taking $\Lambda_{\max } \sim M_{P l}$ yields the standard wrong result, but if instead, we use the expression from eq. (4), we find that

$$
\mathcal{S} \sim A L \mu_{\max }^{3} \sim \frac{N A}{G_{3} L} \sim \frac{A}{G_{N}}
$$

which is nothing but the holographic bound on the number of degrees of freedom in the ultraviolet theory, as it must be if deconstruction is expected to provide an ultraviolet definition of four-dimensional gravity. We conclude that unitarity plus diffeomorphism invariance are sufficient to imply holography. The argument generalizes to an arbitrary number of dimensions.

The infrared theory (i.e., the four-dimensional continuum limit of the lattice theory) lies in the region where the three-dimensional interaction strength is strongly coupled. The cosmological constant problem is to explain why the vacuum energy is small but nonvanishing at long distances in this region of strong coupling.

There are two natural mass scales in the infrared. Each of these arise from the dimensionful parameters in the ultraviolet, the lattice spacing $a$ and the Newton constant $G_{3}$. One scale is simply the four-dimensional Planck mass, $M_{P l}$, which sets the strength of the gravitational interaction. Since $G_{N}=G_{3} a$, the three-dimensional scale is much higher than the effective four-dimensional gravitational scale as we approach the continuum. Thus, $M_{P l}$

\footnotetext{
${ }^{1} \mathrm{UV} / \mathrm{IR}$ mixing also signifies non-locality in the effective action for the Kaluza-Klein modes [9].
} 
is indeed an infrared scale from the three-dimensional point of view. A second low-energy scale, $\Delta m$, is defined by the mass difference between Bose and Fermi excitations in the three-dimensional theory. Given these two scales and the requirement that the vacuum energy vanishes in the limit where the mass splitting between bosonic and fermionic degrees of freedom goes to zero, we can associate a single scale $\omega$ with $M_{P l}$ and $\Delta m$. This scale serves as a cutoff in the computation of the four-dimensional vacuum energy. Dimensional analysis informs us that

$$
\omega \sim \frac{(\Delta m)^{2}}{M_{P l}} .
$$

Since $\omega$ represents the ultraviolet scale in the computation of the vacuum energy density and is determined by infrared quantities $M_{P l}$ and $\Delta m$, this relation is also a manifestation of the UV/IR correspondence.

When evaluating vacuum diagrams in order to estimate the upper bound on the vacuum energy in the infrared, we use $\omega$ as the only effective cutoff in the theory. The naïve expression $^{2}$ for the vacuum energy is bounded by $\omega^{4}$, or

$$
\Lambda \sim M_{P l}^{4}\left(\frac{\Delta m}{M_{P l}}\right)^{8}
$$

Therefore, the observed bound on the vacuum energy density can be realized by a large separation between the mass splitting and the Planck scale.

This argument relies upon a few basic assumptions: dimensional analysis, the UV/IR relation we have discussed previously, three-dimensional supersymmetry, and the notion that the deconstruction of Witten's argument for the vanishing of the cosmological constant in $2+1$ dimensions implies zero vacuum energy at a very low scale set by $\Delta m$. The limit $\Delta m \rightarrow 0$, in which the four-dimensional cosmological constant vanishes, corresponds to the restoration of the mass degeneracy in three dimensions. This observation is consistent with the principle that vanishing dimensionful parameters correspond to enhanced symmetries.

Deconstruction offers a new way of dealing with the famously difficult ultraviolet problems of four-dimensional gravity. In this approach, four-dimensional Lorentz invariance is an emergent symmetry. Deconstruction also leads to a new perspective on the holographic principle as well as on one of the outstanding puzzles of fundamental physics, the cosmological constant problem. The numerical smallness of the cosmological constant is implied by a unique combination of holography and supersymmetry. Given that the total energy density of the universe today is apparently dominated by the energy in the vacuum and the critical rôle supersymmetry has played in the effort to understand the stability of the vacuum, we expect that the deconstruction of four-dimensional gravity will in the future provide many additional insights about Nature.

\footnotetext{
${ }^{2}$ Of course, one should make a careful study of radiative corrections as well, even though these cannot be disastrous if we remember that the vacuum energy is zero, by deconstruction, down to a very low energy scale.
} 


\section{Acknowledgments}

This work is supported in part by the U.S. Department of Energy under contracts DE-FG0291ER40677 (RGL).

\section{References}

[1] G. 't Hooft, arXiv:gr-qc/9310026 L. Susskind, J. Math. Phys. 36, 6377 (1995) arXiv:hep-th/9409089.

[2] J. D. Bekenstein, Phys. Rev. D 7, 2333 (1973); J. M. Bardeen, B. Carter and S. W. Hawking, Commun. Math. Phys. 31, 161 (1973); S. W. Hawking, Commun. Math. Phys. 43, 199 (1975).

[3] J. Maldacena, Adv. Theor. Math. Phys. 2, 231 (1998) arXiv:hep-th/9711200; S. S. Gubser, I. R. Klebanov, A. M. Polyakov, Phys. Lett. B428 (1998) 105 arXiv:hep-th/9802109; E. Witten, Adv. Theor. Math. Phys. 2(1998) 253 arXiv:hep-th/9802150.

[4] C. L. Bennett et al., arXiv:astro-ph/0302207.

[5] A. G. Riess et al. [Supernova Search Team Collaboration], Astron. J. 116, 1009 (1998) arXiv:astro-ph/9805201; S. Perlmutter et al. [Supernova Cosmology Project Collaboration], Astrophys. J. 517, 565 (1999) arXiv:astro-ph/9812133.

[6] C. M. Hull, JHEP 9807, 021 (1998) arXiv:hep-th/9806146; V. Balasubramanian, P. Horava and D. Minic, JHEP 0105, 043 (2001) arXiv:hep-th/0103171; E. Witten, hep-th/0106109; A. Strominger, JHEP 0110, 034 (2001) arXiv:hep-th/0106113; R. Bousso, A. Maloney and A. Strominger, Phys. Rev. D 65, 104039 (2002) arXiv:hep-th/0112218; V. Balasubramanian, J. de Boer and D. Minic, Phys. Rev. D 65, 123508 (2002) arXiv:hep-th/0110108; V. Balasubramanian, J. de Boer and D. Minic, Annals Phys. 303, 59 (2003) arXiv:hep-th/0207245.

[7] N. Arkani-Hamed, A. G. Cohen and H. Georgi, Phys. Rev. Lett. 86, 4757 (2001) arXiv:hep-th/0104005.

[8] E. Witten, Int. J. Mod. Phys. A 10, 1247 (1995) arXiv:hep-th/9409111. See also E. Witten, Mod. Phys. Lett. A 10, 2153 (1995) arXiv:hep-th/9506101.

[9] N. Arkani-Hamed, H. Georgi and M. D. Schwartz, arXiv:hep-th/0210184 N. ArkaniHamed and M. D. Schwartz arXiv:hep-th/0302110.

[10] V. Jejjala, R. G. Leigh and D. Minic, Phys. Lett. B 556, 71 (2003) arXiv:hep-th/0212057.

[11] V. Jejjala, R. G. Leigh and D. Minic, arXiv:hep-th/0302230. 
[12] E. Witten, Nucl. Phys. B 311, 46 (1988); J. H. Horne and E. Witten, Phys. Rev. Lett. 62, 501 (1989). See also A. Achucarro and P. K. Townsend, Phys. Lett. B 180, 89 (1986).

[13] M. Henneaux, Phys. Rev. D 29, 2766 (1984). 